\begin{tabular}{|c|l|}
\hline Title & Partial regularity for a selective smoothing functional for image restoration in BV space \\
\hline Author(s) & Chen, Y unmei; Rao, Murali; Tonegawa, Y oshihiro; Wunderli, T. \\
\hline Citation & Hokkaido University Preprint Series in Mathematics, 693, 1-19 \\
\hline Issue Date & 2005 \\
\hline DOI & 10.14943/83844 \\
\hline Doc URL & http:/hdl.handle.net/2115/69498 \\
\hline Type & bulletin (article) \\
\hline File Information & pre693.pdf \\
\hline
\end{tabular}

Instructions for use 


\title{
PARTIAL REGULARITY FOR A SELECTIVE SMOOTHING FUNCTIONAL FOR IMAGE RESTORATION IN BV SPACE
}

\author{
YUNMEI CHEN, M. RAO, Y. TONEGAWA, T. WUNDERLI
}

\begin{abstract}
.
In this paper we study the partial regularity of a functional on BV space proposed by Chambolle and Lions [3] for the purposes of image restoration. The functional is designed to smooth corrupted images using isotropic diffusion via the Laplacian where the gradients of the image are below a certain threshold $\epsilon$ and retain edges where gradients are above the threshold using the total variation. Here we prove that if the solution $u \in B V$ of the model minimization problem, defined on an open set $\Omega$, is such that the Lebesgue measure of the set where the gradient of $u$ is below the threshold $\epsilon$ is positive, then there exists a non-empty open region $E$ for which $u \in C^{1, \alpha}$ on $E$ and $|\nabla u|<\epsilon$, and $|\nabla u| \geq \epsilon$ on $\Omega \backslash E$ a.e. Thus we indeed have smoothing where $|\nabla u|<\epsilon$.
\end{abstract}

Key words. bounded variation, selective smooothing, image processing, image restoration, noise removal, partial regularity

AMS subject classifications. 49J40, 35K65

1. Introduction. In this paper we investigate the partial regularity for the problem

$$
\min _{u \in B V(\Omega) \cap L^{2}(\Omega)}\left\{\int_{\Omega} \varphi(D u)+\frac{1}{2} \int_{\Omega}(u-I)^{2} d x\right\}
$$

where $\varphi$ is the following $C^{1}$ convex function defined on $\mathbf{R}^{n}$

$$
\varphi(p)= \begin{cases}\frac{1}{2}|p|^{2} & \text { if }|p|<1 \\ |p|-\frac{1}{2} & \text { if }|p| \geq 1\end{cases}
$$

$\Omega \subset \mathbf{R}^{n}$ is a bounded domain with Lipschitz boundary, and $I \in L^{\infty}(\Omega) \cap B V(\Omega)$ is given. This functional has been proposed for use in image restoration in [3]. For problems of image restoration, we consider an image to be a real valued function defined on an open rectangle $\Omega \subset \mathbf{R}^{n}$. We are then given an image $I$ corrupted by noise, that is,

$$
I=u_{\text {original }}+\eta
$$

where $u_{\text {original }}$ is the true image and $\eta$ is noise. We thus want to recover $u_{\text {original }}$ as much as possible from the given $I$.

TV-based diffusion for image restoration was introduced in [13] as a method of preserving features while removing noise (see also $[2,3,15,12]$ ). The definition of the total variation seminorm for $u \in L^{1}(\Omega)$, given by

$$
T V(u)=\sup \left\{\int_{\Omega} u \operatorname{div}(\varphi) d x: \varphi \in C_{0}^{1}\left(\Omega, \mathbf{R}^{n}\right),|\varphi| \leq 1\right\},
$$

does not require differentiability or even continuity of $u$. Thus images with discontinuities are allowed as solutions in the space of $B V(\Omega)$, which is the space of the functions $u \in L^{1}(\Omega)$ with $T V(u)<\infty$. In [3] the restored image is taken to be the 
minimizer of a combination of the total variation and the squared $L^{2}(\Omega)$ norm of the gradient. That is, we minimize

$$
\frac{1}{2 a} \int_{|\nabla u|<a}|\nabla u|^{2} d x+\int_{|\nabla u| \geq a}\left(|\nabla u|-\frac{a}{2}\right)+\frac{1}{2} \int_{\Omega}(u-I)^{2} d x .
$$

Using the above functional we then expect to have isotropic diffusion where the image is more uniform $(|\nabla u|<a)$, and feature preservation via TV-based diffusion where the boundaries of features are present (the locations where the image gradients most likely have high magnitude: $|\nabla u| \geq a$ ). Without loss of generality we take $a=1$ as in (1.1).

For $u \in B V(\Omega)$ the gradient of $u$ is a measure $D u$; it can be decomposed into its absolutely continuous and singular parts with respect to Lebesgue measure, that is

$$
D u=\nabla u d x+D^{s} u .
$$

See [5] for a complete discussion. Then we define ([8])

$$
J(u) \equiv \int_{\Omega} \varphi(D u) \equiv \int_{\Omega} \varphi(\nabla u) d x+\int_{\Omega}\left|D^{s} u\right|
$$

with

$$
\int_{\Omega}\left|D^{s} u\right| \equiv \int_{\Omega} d\left|D^{s} u\right|=\left|D^{s} u\right|(\Omega) .
$$

It is important to note $([16]$ or $[8])$ that the functional $J$ can also be written as

$$
J(u)=\sup _{\phi \in C_{0}^{1}\left(\Omega, \mathbf{R}^{n}\right)}\left\{-\int_{\Omega}\left(\frac{1}{2}|\phi|^{2}+u \operatorname{div}(\phi)\right) d x:|\phi(x)| \leq 1 \forall x \in \Omega\right\} .
$$

Using this, we see that the functional $J$ is lower semicontinuous with respect to convergence in $L^{1}(\Omega)$. Then by a standard argument we can show that there is a unique solution $u \in B V(\Omega) \cap L^{2}(\Omega)$ to (1.1). Now we are interested as to whether or not this solution $u \in B V$ is smooth on the region where $|\nabla u|<1$. If so, it shows that the denoising governed by (1.1) smoothes out lower gradients while preserving the boundaries of features, which are the discontinuities in an image.

We now state the two main partial regularity results of this paper.

TheOREm 1.1. \& If $u$ is the solution to (1.1), then for any given $0<\mu<1$ there exist positive constants $\epsilon_{0}$ and $\kappa_{0}$ depending only on $n$ and $\mu$ such that if

$$
\frac{1}{\left|B_{r}\right|} \int_{B_{r}(a)}|D u-l| \leq \epsilon_{0}
$$

holds for some ball $B_{r}(a) \subset \subset \Omega$ and for some $l \in \mathbf{R}^{n}$, with

$$
r C\left(1+\|I\|_{L^{\infty}(\Omega)}\right)<\kappa_{0} \quad \text { and } \quad|l|<1-2 \mu,
$$

for some constant $C$ depending only on $n$ and $\Omega$ then,

$$
\left|D^{s} u\right|\left(B_{r / 2}(a)\right)=0 \quad \text { and } \quad|\nabla u|<1-\mu \quad \text { on } \quad B_{r / 2}(a)
$$

and $u$ solves

$$
-\Delta u=I-u \quad \text { on } \quad B_{r / 2}(a)
$$


Hence $u \in C^{1, \alpha}\left(B_{r / 2}(a)\right)$ for any $\alpha<1$.

Theorem 1.2. \& Let $u$ be as in Theorem (1.1). If $\mathcal{L}^{n}(\{|\nabla u|<1\})>0$, then there exists a nonempty open region $E$ on which $u$ is $C^{1, \alpha},|\nabla u|<1$ and $u$ solves

$$
-\Delta u=I-u \quad \text { on } \quad E .
$$

In addition we have $|\nabla u| \geq 1$ a.e. on $\Omega \backslash E$.

It is actually straightforward to show that Theorem 1.2 is a direct consequence of Theorem 1.1. Thus from Theorem 1.2, we do indeed have smoothing where $|\nabla u|<1$.

Here we should point out that partial regularity results were obtained in [1] for minimizers in $B V(\Omega)$ of functionals of the form $\int_{\Omega}(F(x, D u)+G(x, u))$ where $F(x, p)$ is a convex function in $p$ with $c_{1}|p| \leq F(x, p) \leq c_{2}(1+|p|)$ for all $p \in \mathbf{R}^{n}, F$ is locally Hölder continuous in $x$, and $G(x, z)$ satisfies Hölder continuity conditions in both $x$ and $z$. In our case, $G(x, z)=1 / 2(z-I(x))^{2}$ with only the stated assumption on $I$, and therefore their results can not directly be applied in our case. Moreover, our approach is quite different from theirs, and can be applied to more general cases.

The partial regularity results for the flow associated with the minimization problem (1) is also discussed in [11] for more general $\varphi$. However, these hold only $\Omega \subset \mathbf{R}^{n}$ for $n=1$ and $n=2$. We also apply some different techniques to get our results.

2. Proof of Theorem 1.1 and Theorem 1.2. First we will show that the solution $u$ to $(1.1)$ is in $L^{\infty}(\Omega)$. To prove this we could consider the time evolution problem corresponding to (1.1), prove an $L^{\infty}$ bound for the time dependent solution $u(x, t)$, and then consider the time asymptotic limit $u$, which is the solution to (1.1). We would then conclude that $u \in L^{\infty}(\Omega)$. The following, however, provides a proof of this without having to consider the time evolution of (1.1).

LEMMA 2.1. If $u$ is the solution to (1.1), then $u \in L^{\infty}(\Omega)$. In fact, we have $\|u\|_{L^{\infty}(\Omega)} \leq\|I\|_{L^{\infty}(\Omega)}$.

Proof. Let $\varphi_{\epsilon}$ be defined on $\mathbf{R}^{n}$ by

$$
\varphi_{\epsilon}(p)= \begin{cases}\frac{1}{2}|p|^{2} & \text { if }|p|<1 \\ \frac{1}{1+\epsilon}|p|^{1+\epsilon}+\left(\frac{1}{2}-\frac{1}{1+\epsilon}\right) & \text { if }|p| \geq 1\end{cases}
$$

for $\epsilon>0$, and consider the following minimization problem:

$$
\min _{u \in W^{1,1+\epsilon}(\Omega) \cap L^{2}(\Omega)}\left\{\int_{\Omega} \varphi_{\epsilon}(\nabla u)+\frac{1}{2} \int_{\Omega}(u-I)^{2} d x\right\} .
$$

By standard methods, there is a unique solution $u_{\epsilon}$ to this problem. We follow a standard truncation argument where we fix $\epsilon$ and $t \geq 0$ and let $v=\min \left(u_{\epsilon}, t\right)$. Noting that $v \in W^{1,1+\epsilon}(\Omega) \cap L^{2}(\Omega)$ with

$$
\nabla v= \begin{cases}\nabla u_{\epsilon} & \text { if } u_{\epsilon}<t \\ 0 & \text { if } u_{\epsilon} \geq t\end{cases}
$$

we have

$$
\int_{\Omega} \varphi_{\epsilon}\left(\nabla u_{\epsilon}\right)+\frac{1}{2} \int_{\Omega}\left(u_{\epsilon}-I\right)^{2} d x \leq \int_{\Omega} \varphi_{\epsilon}(\nabla v)+\frac{1}{2} \int_{\Omega}(v-I)^{2} d x
$$


and thus after subtracting

$$
\int_{\left\{u_{\epsilon} \geq t\right\}} \varphi_{\epsilon}\left(\nabla u_{\epsilon}\right) d x+\int_{\left\{u_{\epsilon} \geq t\right\}}\left(u_{\epsilon}-I\right)^{2} d x \leq \int_{\left\{u_{\epsilon} \geq t\right\}}(t-I)^{2} d x .
$$

Hence

$$
\int_{\left\{u_{\epsilon} \geq t\right\}}\left(u_{\epsilon}-I\right)^{2} d x \leq \int_{\left\{u_{\epsilon} \geq t\right\}}(t-I)^{2} d x .
$$

But setting $t=\|I\|_{L^{\infty}(\Omega)}$ we see that if ess sup $u_{\epsilon}>t$ then

$$
\int_{\left\{u_{\epsilon} \geq t\right\}}(t-I)^{2} d x<\int_{\left\{u_{\epsilon} \geq t\right\}}\left(u_{\epsilon}-I\right)^{2} d x
$$

which contradicts the above, hence ess $\sup u_{\epsilon} \leq\|I\|_{L^{\infty}(\Omega)}$. Applying a similar argument to $v=\max \left(u_{\epsilon},-t\right)$ for $t=\|I\|_{L^{\infty}(\Omega)}$ we get ess inf $u_{\epsilon} \geq-\|I\|_{L^{\infty}(\Omega)}$ and thus $\left\|u_{\epsilon}\right\|_{L^{\infty}(\Omega)} \leq\|I\|_{L^{\infty}(\Omega)}$. Furthermore, letting $v=0$ in (2.1) we see that $u_{\epsilon}$ is bounded in $W^{1,1+\epsilon}(\Omega) \cap L^{2}(\Omega) \subset B V(\Omega) \cap L^{2}(\Omega)$ independent of $\epsilon$. Thus there is a $\tilde{u} \in B V(\Omega) \cap L^{2}(\Omega)$ and a subsequence of $\left\{u_{\epsilon}\right\}$, still denoted by $\left\{u_{\epsilon}\right\}$, such that $u_{\epsilon} \rightarrow \tilde{u}$ strongly in $L^{1}(\Omega), u_{\epsilon} \rightarrow \tilde{u}$ weakly in $L^{2}(\Omega)$, and $u_{\epsilon} \rightarrow \tilde{u}$ a.e in $\Omega$. Letting $\epsilon \rightarrow 0$ in (2.1), noting that $\varphi(p) \leq \varphi_{\epsilon}(p)$ for all $p, \int_{\Omega} \varphi_{\epsilon}(\nabla v) \rightarrow \int_{\Omega} \varphi(\nabla v)$, lower semicontinuity of the functional $\int_{\Omega} \varphi(\nabla u)$ defined on $B V(\Omega)$, and weak lower semicontinuity of the second term on the left hand side, we get

$$
\int_{\Omega} \varphi(\nabla \tilde{u})+\frac{1}{2} \int_{\Omega}(\tilde{u}-I)^{2} d x \leq \int_{\Omega} \varphi(\nabla v)+\frac{1}{2} \int_{\Omega}(v-I)^{2} d x
$$

for all $v \in W^{1,1+\epsilon}(\Omega) \cap L^{2}(\Omega)$. We now note ([8]) that for any $v \in B V(\Omega) \cap L^{2}(\Omega)$ there exists a sequence $v_{n}$ in $C^{\infty}(\bar{\Omega})$ such that

$$
\int_{\Omega} \varphi\left(\nabla v_{n}\right) d x \rightarrow \int_{\Omega} \varphi(\nabla v)
$$

and $v_{n} \rightarrow v$ in $L^{1}(\Omega)$, and since $v \in L^{2}(\Omega)$ from the construction of $v_{n}$ ([8]) we can also take $v_{n} \rightarrow v$ in $L^{2}(\Omega)$. Therefore we see that the above holds for all $v \in$ $B V(\Omega) \cap L^{2}(\Omega)$ as well. Hence $\tilde{u}$ solves (1.1). By uniqueness, $\tilde{u}=u$. By the uniform $L^{\infty}$ bound for $u_{\epsilon}$ and the convergence of $u_{\epsilon}$ to $u$ a.e. in $\Omega$ we have $u \in L^{\infty}(\Omega)$ with $\|u\|_{L^{\infty}(\Omega)} \leq\|I\|_{L^{\infty}(\Omega)}$ प

Throughout the rest of the paper, we fix $\mu>0$ and unless otherwise stated, all constants depend at most on $n, \mu, u, \Omega, \varphi$, and possibly $I$.

We begin with a local lower bound estimate for any $B V$ function $u$ and $C^{1}$ function $h$ with gradient strictly less than 1 .

Lemma 2.2. Let $u \in B V\left(B_{r}(a)\right)$ for $B_{r}(a) \subset \subset \Omega$ and $h \in C^{1}\left(\bar{B}_{r}(a)\right)$ with

$$
\sup _{B_{r}(a)}|\nabla h| \leq 1-\mu,
$$

then

$$
\int_{B_{r}(a)} \varphi(D u)-\int_{B_{r}(a)} \varphi(\nabla h) d x \geq \mu \int_{B_{r}(a)}\left|D^{s} u\right|+\int_{B_{r}(a)} \nabla(u-h) \cdot \nabla h d x
$$




$$
\begin{gathered}
+\int_{B_{r}(a)} D^{s} u \cdot \nabla h+\frac{\mu^{2}}{2} \int_{B_{r}(a) \cap\{|\nabla u| \geq 1\}}|\nabla u| d x \\
+\frac{1}{2} \int_{B_{r}(a) \cap\{|\nabla u|<1\}}|\nabla(u-h)|^{2} d x .
\end{gathered}
$$

Proof. Where $|\nabla u| \geq 1$, we have

$$
\begin{gathered}
\varphi(\nabla u)-\varphi(\nabla h)-\nabla(u-h) \cdot \nabla h \\
=|\nabla u|-\frac{1}{2}+\frac{1}{2}|\nabla h|^{2}-\nabla u \cdot \nabla h \\
\geq \frac{1}{2}\left(2|\nabla u|-1-2|\nabla u||\nabla h|+|\nabla h|^{2}\right) \\
=\frac{1}{2}(2|\nabla u|-1-|\nabla h|)(1-|\nabla h|) \geq \frac{\mu^{2}}{2}|\nabla u| .
\end{gathered}
$$

Where $|\nabla u|<1$, we have

$$
\varphi(\nabla u)-\varphi(\nabla h)-\nabla(u-h) \cdot \nabla h=\frac{1}{2}|\nabla(u-h)|^{2} .
$$

We now obtain the lemma by using

$$
\int_{B_{r}(a)}\left|D^{s} u\right| \geq \int_{B_{r}(a)} D^{s} u \cdot \nabla h+\int_{B_{r}(a)}\left|D^{s} u\right|(1-|\nabla h|),
$$

the assumption on $h$, and the above estimates.

We now fix $B_{2 r}(a) \subset \subset \Omega$. Let $v$ be a Lipschitz function defined on $B_{2 r}(a)$ and assume there exists an $l \in \mathbf{R}^{n}$ with $|l| \leq 1-2 \mu$, such that $\sup _{B_{2 r}(a)}|\nabla v-l| \leq \beta^{2 \delta}$ for $\delta>0$ and $0<\beta<1$ to be chosen later. Also let $\bar{v}$ be defined by $\bar{v}(x)=v(x)-l \cdot x$. Let $\eta_{\epsilon}$ be the usual mollifier on $\mathbf{R}^{n}$ and denote $\bar{v}_{\beta}=\eta_{r \beta} * \bar{v}$ and $v_{\beta}=\eta_{r \beta} * v$. We have the following estimates from [14]:

$$
\begin{gathered}
\sup _{B_{r}(a)}\left|\nabla v_{\beta}-l\right|=\sup _{B_{r}(a)}\left|\nabla \bar{v}_{\beta}\right| \leq \beta^{2 \delta}, \\
\sup _{B_{r}(a)}\left|v_{\beta}-v\right|=\sup _{B_{r}(a)}\left|\bar{v}_{\beta}-\bar{v}\right| \leq r \beta \sup _{B_{r}(a)}\left|\nabla \bar{v}_{\beta}\right| \leq r \beta^{1+2 \delta}, \\
r^{\delta} \sup _{B_{r}(a)}|x-y|^{-\delta}\left|\nabla v_{\beta}(x)-\nabla v_{\beta}(y)\right| \\
\leq c_{1} r^{\delta} \sup _{B_{r}(a)}|\nabla v-l| \sup _{x^{\prime} \neq y^{\prime}}\left|x^{\prime}-y^{\prime}\right|^{-\delta}\left|\eta_{1}\left((r \beta)^{-1} x^{\prime}\right)-\eta_{1}\left((r \beta)^{-1} y^{\prime}\right)\right| \\
\leq c_{2} \beta^{2 \delta} \beta^{-\delta}=c_{2} \beta^{\delta} .
\end{gathered}
$$


Now for any $\tilde{r} \in\left[\frac{r}{2}, r\right]$ there exists a unique solution $([7]) w \in H^{1}\left(B_{\tilde{r}}(a)\right) \cap C^{1, \delta}\left(\bar{B}_{\tilde{r}}(a)\right)$ with $\delta \in(0,1)$ for the problem

$$
-\Delta w=I-w \quad \text { on } B_{\tilde{r}}(a), \quad w=v_{\beta} \quad \text { on } \partial B_{\tilde{r}}(a) .
$$

Lemma 2.3. For $I \in L^{\infty}(\Omega)$, the solution $w$ to (2.5) satisfies

$$
\|w\|_{L^{\infty}\left(B_{\tilde{r}}(a)\right)} \leq\left\|v_{\beta}\right\|_{L^{\infty}\left(\partial B_{\tilde{r}}(a)\right)}+\|I\|_{L^{\infty}(\Omega)} .
$$

(2.7) $\sup _{B_{\tilde{r}(a)}}|\nabla w-l| \leq c_{3}\left(\beta^{\delta}+r\left(\|I\|_{L^{\infty}(\Omega)}+\left\|v_{\beta}\right\|_{L^{\infty}\left(\partial B_{\tilde{r}}(a)\right)}\right)\right), \quad$ for any $l \in R^{n}$.

$$
\begin{gathered}
\sup _{x, y \in B_{\tilde{r} / 2}(a)} \frac{|\nabla w(x)-\nabla w(y)|}{|x-y|^{1 / 2}} \leq c_{4}\left(\frac{1}{r^{n+1 / 2}} \int_{\left.\partial B_{\tilde{r}}(a)\right)}\left|v_{\beta}\right| d \mathcal{H}^{n-1}\right. \\
\left.+r^{1 / 2}\left(\|I\|_{L^{\infty}(\Omega)}+\left\|v_{\beta}\right\|_{L^{\infty}\left(\partial B_{\tilde{r}}(a)\right)}\right)\right) .
\end{gathered}
$$

Proof. The estimate (2.6) is from Theorem 8.16 in [7]. To prove (2.7) and (2.8), we decompose $w$ as $w=w_{1}+w_{2}$, such that

$$
-\Delta w_{1}=I-w \quad \text { on } B_{\tilde{r}}(a), \quad w_{1}=0, \quad \text { on } \partial B_{\tilde{r}}(a) .
$$

and

$$
-\Delta w_{2}=0 \quad \text { on } B_{\tilde{r}}(a), \quad w=v_{\beta} \quad \text { on } \partial B_{\tilde{r}}(a) .
$$

Let $\tilde{w}_{2} \equiv w_{2}-v_{\beta}$. Then $\tilde{w}_{2}$ solves

$$
-\Delta \tilde{w}_{2}=-\operatorname{div}\left(\nabla v_{\beta}-l\right) \quad \text { on } B_{\tilde{r}}(a), \quad \tilde{w}_{2}=0 \quad \text { on } \partial B_{\tilde{r}}(a),
$$

for any $l \in R^{n}$. Representing the solution of (2.9) using Green's function, i.e., $w_{1}(x)=$ $\int_{B_{\tilde{r}}(a)} \Gamma(x-y)(I-w)(y) d y$, where $\Gamma$ is the fundamental solution of Laplace's equation, it is not difficult to get

$$
\left\|\nabla w_{1}\right\|_{L^{\infty}\left(B_{\tilde{r}}(a)\right)} \leq c r\|I-w\|_{L^{\infty}\left(B_{\tilde{r}}(a)\right)}
$$

where $c$ is independent of $r$.

Moreover, by Sobolev imbedding theorem, Theorem 9.9 in [7], and (2.6),

$$
\begin{aligned}
& \left\|\nabla w_{1}\right\|_{C^{0,1 / 2}\left(B_{\tilde{r}}(a)\right)} \leq c\left\|w_{1}\right\|_{W^{2,2 n}\left(B_{\tilde{r}}(a)\right)} \leq c\|I-w\|_{L^{2 n}\left(B_{\tilde{r}}(a)\right)} \\
& \leq c r^{1 / 2}\|I-w\|_{L^{\infty}\left(B_{\tilde{r}}(a)\right)} \leq c r^{1 / 2}\left(\left\|v_{\beta}\right\|_{L^{\infty}\left(\partial B_{\tilde{r}}(a)\right)}+\|I\|_{L^{\infty}(\Omega)}\right) .
\end{aligned}
$$

Next we shall estimate $w_{2}$. Multiplying by $\tilde{w}_{2}$ to the both sides of $(2.11)$ and integrating over $B_{\tilde{r}}(a)$, by a simple computation and using (2.2), one can have for any $l \in R^{n}$,

$$
\int_{B_{\tilde{r}}(a)}\left|\nabla w_{2}-l\right|^{2} d x \leq c \int_{B_{\tilde{r}}(a)}\left|\nabla v_{\beta}-l\right|^{2} d x \leq c r^{n} \beta^{4 \delta}
$$


where $c>0$ is a constant independent of $r$.

Furthermore, applying Theorem 8.16 and 8.33 (with a rescaling argument) in [7] to (2.11), we get the following estimates:

$$
\left\|\tilde{w}_{2}\right\|_{L^{\infty}\left(B_{\tilde{r}}\right)} \leq c\left\|\nabla v_{\beta}-l\right\|_{L^{\infty}\left(B_{\tilde{r}}\right)},
$$

and

$(2.16) r^{\delta}\left[D \tilde{w}_{2}\right]_{C^{0, \delta}\left(B_{\tilde{r}}\right)} \leq c\left(\left\|\tilde{w}_{2}\right\|_{L^{\infty}\left(B_{\tilde{r}}\right)}+\left\|\nabla v_{\beta}-l\right\|_{L^{\infty}\left(B_{\tilde{r}}\right)}+r^{\delta}\left[D v_{\beta}\right]_{C^{0, \delta}\left(B_{\tilde{r}}\right)}\right)$,

where $c>0$ is a constant independent of $r$. Inserting (2.15) into (2.16), and using (2.2) and (2.4), it yields

$$
\begin{gathered}
r^{\delta}\left[D w_{2}\right]_{C^{0, \delta}\left(B_{\tilde{r}}\right)} \leq\left(r^{\delta}\left[D \tilde{w}_{2}\right]_{C^{0, \delta}\left(B_{\tilde{r}}\right)}+r^{\delta}\left[D v_{\beta}\right]_{C^{0, \delta}\left(B_{\tilde{r}}\right)}\right) \\
\leq c\left(\left\|\nabla v_{\beta}-l\right\|_{L^{\infty}\left(B_{\tilde{r}}\right)}+r^{\delta}\left[D v_{\beta}\right]_{C^{0, \delta}\left(B_{\tilde{r}}\right)}\right) \leq c \beta^{\delta} .
\end{gathered}
$$

Now we can estimate $\sup _{B_{\tilde{r}}(a)}\left|\nabla w_{2}-l\right|$. Denoting $\left|B_{\tilde{r}}(a)\right|^{-1} \int_{B_{\tilde{r}}(a)} f d x$ by $(f)_{B_{\tilde{r}}(a)}$, and using (2.14) and (2.17), we get

$$
\begin{aligned}
& \sup _{B_{\tilde{r}}(a)}\left|\nabla w_{2}-l\right| \leq \sup _{B_{\tilde{r}}(a)}\left\{\left|\nabla w_{2}-\left(\nabla w_{2}\right)_{B_{\tilde{r}}(a)}\right|+\left|\left(\nabla w_{2}\right)_{B_{\tilde{r}}(a)}-l\right|\right\} \\
& \leq r^{\delta}\left[D w_{2}\right]_{C^{0, \delta}\left(B_{\tilde{r}}\right)}+\left|B_{\tilde{r}}(a)\right|^{-1 / 2}\left(\int_{B_{\tilde{r}(a)}}\left|\nabla w_{2}-l\right|^{2}\right)^{1 / 2} d x \leq c \beta^{\delta},
\end{aligned}
$$

here we used (2.14) and (2.17) in the last inequality.

We then have, from (2.6) and (2.18),

$$
\begin{aligned}
& \sup _{B_{\tilde{r}}(a)}|\nabla w-l| \leq \sup _{B_{\tilde{r}}(a)}\left|\nabla w_{2}-l\right|+\sup _{B_{\tilde{r}}(a)}\left|\nabla w_{1}\right| \\
& \leq c_{3}\left(\beta^{\delta}+r\left(\|I\|_{L^{\infty}\left(B_{\tilde{r}}(a)\right)}+\left\|v_{\beta}\right\|_{L^{\infty}\left(\partial B_{\tilde{r}}(a)\right)}\right)\right) .
\end{aligned}
$$

(2.7) is proved. To prove (2.8) we represent $w_{2}$ by the Poisson's formula on the ball $B_{\tilde{r}}(a)$, i.e.

$$
w_{2}(x)=\frac{\tilde{r}^{2}-|x|^{2}}{n \alpha_{n} r} \int_{\partial B_{\tilde{r}}(a)} \frac{v_{\beta}(y)}{|x-y|^{n}} d S_{y}, \quad x \in B_{\tilde{r}}(a),
$$

where $\alpha_{n}$ represents the volume of $n$ dimensional unit ball. A direct computation leads to the estimate:

$$
\sup _{B_{\tilde{r} / 2}(a)}\left|D^{2} w_{2}\right| \leq c r^{-n-1} \int_{\partial B_{\tilde{r}}(a)}\left|v_{\beta}(y)\right| d S_{y},
$$

where $c>0$ dependents only on $n$. Then we have

$$
\begin{gathered}
\sup _{x, y \in B_{\tilde{r} / 2}(a)} \frac{\left|\nabla w_{2}(x)-\nabla w_{2}(y)\right|}{|x-y|^{1 / 2}} \leq\left(\sup _{x, y \in B_{\tilde{r} / 2}(a)}\left|D^{2} w_{2}\right|\right)|x-y|^{1 / 2} \\
\leq \frac{c}{r^{n+1 / 2}} \int_{\left.\partial B_{\tilde{r}}(a)\right)}\left|v_{\beta}\right| d \mathcal{H}^{n-1} .
\end{gathered}
$$


Now (2.8) follows from (2.13) and (2.19) imediately.

LEMma 2.4. Suppose there is a $v \in C^{0,1}\left(B_{2 r}(a)\right)$ and $l \in \mathbf{R}^{n}$ with $|l| \leq 1-2 \mu$, $\sup _{B_{2 r}(a)}|\nabla v-l| \leq \beta^{2 \delta}$, and $\sup _{B_{2 r}(a)}|v| \leq C_{u}$ where $C_{u}$ is a constant depending only on $u$. Let $v_{\beta}, \tilde{r}$, and $w$ be as in the previous discussion. Then there exists constants $c_{5}$ and $c_{6}$ such that if $\beta \leq c_{5}$ and $r\left(C_{u}+\|I\|_{L^{\infty}(\Omega)}\right) \leq c_{6}$ then

$$
\begin{gathered}
\int_{B_{\tilde{r}}(a)} \varphi(D u)-\int_{B_{\tilde{r}}(a)} \varphi(\nabla w) d x \geq \int_{\partial B_{\tilde{r}}(a)}\left(u-v_{\beta}\right) \frac{\partial w}{\partial n} d \mathcal{H}^{n-1} \\
+\int_{B_{\tilde{r}}(a)}(u-w)(I-w) d x+\mu \int_{B_{\tilde{r}}(a)}\left|D^{s} u\right|+\frac{\mu^{2}}{2} \int_{B_{\tilde{r}}(a) \cap\{|\nabla u| \geq 1\}}|\nabla u| d x \\
+\frac{1}{2} \int_{B_{\tilde{r}}(a) \cap\{|\nabla u| \leq 1\}}|\nabla(u-w)|^{2} d x \\
\geq \int_{\partial B_{\tilde{r}}(a)}\left(u-v_{\beta}\right) \frac{\partial w}{\partial n} d \mathcal{H}^{n-1}+\frac{1}{2} \int_{B_{\tilde{r}}(a)}(w-I)^{2} d x-\frac{1}{2} \int_{B_{\tilde{r}}(a)}(u-I)^{2} d x \\
+\mu \int_{B_{\tilde{r}}(a)}\left|D^{s} u\right|+\frac{\mu^{2}}{2} \int_{B_{\tilde{r}}(a) \cap\{|\nabla u| \geq 1\}}|\nabla u| d x \\
+\frac{1}{2} \int_{B_{\tilde{r}}(a) \cap\{|\nabla u|<1\}}|\nabla(u-w)|^{2} d x
\end{gathered}
$$

Proof. ¿From (2.7)-(2.8), the definition of $v_{\beta}$, and the assumption on $l$ we see that

$$
\begin{gathered}
\sup _{B_{\tilde{r}(a)}}|\nabla w| \leq \sup _{B_{\tilde{r}(a)}}|\nabla w-l|+|l| \\
\leq c_{3}\left(\beta^{\delta}+r\left(\|v\|_{L^{\infty}\left(\partial B_{\tilde{r}}(a)\right)}+\|I\|_{L^{\infty}(\Omega)}\right)\right)+1-2 \mu \\
\leq c_{3}\left(\beta^{\delta}+r\left(C_{u}+\|I\|_{L^{\infty}(\Omega)}\right)\right)+1-2 \mu .
\end{gathered}
$$

Later, $v$ will be chosen (see for instance [10]) to be a Lipschitz approximation of $u$ so that $\|v\|_{L^{\infty}\left(B_{2 r}(a)\right)}$ can be bounded by a constant $C_{u}$ depending only on $u$. Now choose $c_{5}$ and $c_{6}$ such that $\beta^{\delta} \leq c_{5}$ and

$$
r\left(C_{u}+\|I\|_{L^{\infty}(\Omega)}\right) \leq c_{6}
$$

imply

$$
c_{3}\left(\beta^{\delta}+r\left(C_{u}+\|I\|_{L^{\infty}(\Omega)}\right)\right) \leq \mu
$$


Thus

$$
\sup _{B_{\tilde{r}(a)}}|\nabla w| \leq 1-\mu .
$$

The conditions of Lemma 2.2 now hold for $h=w$. Substituting in $w$ for $h$ in the inequality in Lemma 2.2, integrating by parts, and using Young's inequality for $(u-$ $w)(I-w)=-(u-I)(w-I)+(I-w)^{2}$ the Lemma is proved. $\square$

LEMMA 2.5. If the function $u \in B V(\Omega)$ is solution to (1.1), then

$$
\begin{aligned}
& \int_{B_{r}} \varphi(D u)-\int_{B_{r}} \varphi(D w) \leq 1 / 2 \int_{B_{r}}(w-I)^{2} d x \\
& -1 / 2 \int_{B_{r}}(u-I)^{2} d x+\int_{\partial B_{r}}|T w-T u| d \mathcal{H}^{n-1}
\end{aligned}
$$

for any $w \in B V\left(B_{r}\right), B_{r} \subset \subset \Omega$. Here $T$ denotes the trace operator on $B V$.

Proof. Let $w \in B V\left(B_{r}\right)$ and define

$$
\zeta= \begin{cases}w-u & \text { on } B_{r} \\ 0 & \text { in } \Omega \backslash \bar{B}_{r} .\end{cases}
$$

Then since $u$ is a solution we have letting $v=u+\zeta$ in (1.1) and using Theorem 1 of section 5.4 in $[5]$

$$
\begin{gathered}
\int_{\Omega} \varphi(D u)+1 / 2 \int_{\Omega}(u-I)^{2} d x \leq \int_{B_{r}} \varphi(D w)+\int_{\partial B_{r}}|T w-T u| d \mathcal{H}^{n-1} \\
\quad+\int_{\Omega \backslash \bar{B}_{r}} \varphi(D u)+1 / 2 \int_{B_{r}}(w-I)^{2} d x+1 / 2 \int_{\Omega \backslash B_{r}}(u-I)^{2} d x .
\end{gathered}
$$

Hence

$$
\begin{gathered}
\int_{\bar{B}_{r}} \varphi(D u)+1 / 2 \int_{B_{r}}(u-I)^{2} d x \leq \int_{B_{r}} \varphi(D w)+1 / 2 \int_{B_{r}}(w-I)^{2} d x \\
+\int_{\partial B_{r}}|T w-T u| d \mathcal{H}^{n-1} .
\end{gathered}
$$

$\square$ We use the above lemma, Lemma 2.4, and estimates (2.2)-(2.4) to obtain the following inequality for the solution $u$ to (1.1):

LEMma 2.6. Let $v, l$ be as in Lemma 2.4 with

$$
r\left(C_{u}+\|I\|_{L^{\infty}(\Omega)}\right) \leq c_{6},
$$

$w$ as in (2.5), and $u$ a solution to (1.1). Then

$$
\begin{aligned}
& \int_{B_{\tilde{r}}(a)}\left|D^{s} u\right|+\int_{B_{\tilde{r}(a)} \cap\{|\nabla u| \geq 1\}}|\nabla u| d x+\int_{B_{\tilde{r}(a)} \cap\{|\nabla u|<1\}}|\nabla(u-w)|^{2} d x \\
& \leq c_{7} \int_{\partial B_{\tilde{r}(a)}}|u-v| d \mathcal{H}^{n-1}+c_{8} r^{n} \beta^{1+2 \delta} .
\end{aligned}
$$


where $u$ and $v$ on $\partial B_{\tilde{r}}(a)$ is understood in the sense of trace.

Proof. By the previous lemma with $w$ from (2.5) and Lemma 2.4 we have

$$
\begin{aligned}
& \int_{\partial B_{\tilde{r}(a)}}\left|u-v_{\beta}\right| d \mathcal{H}^{n-1} \geq \int_{B_{\tilde{r}(a)}} \varphi(D u)+\frac{1}{2} \int_{B_{\tilde{r}}(a)}(u-I)^{2} d x \\
& -\int_{B_{\tilde{r}}(a)} \varphi(\nabla w) d x-\frac{1}{2} \int_{B_{\tilde{r}}(a)}(w-I)^{2} d x \\
& \geq \int_{\partial B_{\tilde{r}(a)}}\left(u-v_{\beta}\right) \frac{\partial w}{\partial n} d \mathcal{H}^{n-1}+\mu \int_{B_{\tilde{r}(a)}}\left|D^{s} u\right|+\frac{\mu^{2}}{2} \int_{B_{\tilde{r}(a)} \cap\{|\nabla u| \geq 1\}}|\nabla u| d x \\
& +\frac{1}{2} \int_{B_{\tilde{r}(a)} \cap\{|\nabla u|<1\}}|\nabla(u-w)|^{2} d x .
\end{aligned}
$$

The lemma is thus proved by using (2.20) and the estimate for $\left|v-v_{\beta}\right|$ from (2.3).

We have the following first variational formula from Hardt and Kinderlehrer [8]: if $u$ is a solution to (1.1)

$$
\int_{\Omega} \sigma \cdot \nabla \zeta d x+\int_{\Omega} \sigma \cdot \xi\left|D^{s} u\right|=-\int_{\Omega}(u-I) \zeta d x
$$

where $\zeta$ is any function in $B V_{0}(\Omega)$ with $D^{s} \zeta<<\left|D^{s} u\right|, \xi$ is the Radon-Nikodym derivative of $D^{s} \zeta$ with respect to $\left|D^{s} u\right|$, and $\sigma \in L^{1}(\Omega)$ is the stress tensor defined by

$$
\sigma(u)= \begin{cases}\varphi_{P}(\nabla u) & \text { in } \Omega_{a} \\ D^{s} u /\left|D^{s} u\right| & \text { in } \Omega_{s}\end{cases}
$$

Here $D^{s} u /\left|D^{s} u\right|$ denotes the Radon-Nikodym derivative of $D^{s} u$ with respect to $\left|D^{s} u\right|$ and $\Omega=\Omega_{a} \cup \Omega_{s}$ is the decomposition of $\Omega$ with respect to the mutually singular measures $\mathcal{L}^{n}$ and $\left|D^{s} u\right|$. Clearly $|\sigma(u)| \leq 1$. Note that $\sigma(u)$ depends only on $u$. In the sequel we will write $\sigma$ instead of $\sigma(u)$ and write the left hand side of (2.21) as

$$
\int_{\Omega} \sigma \cdot D \zeta
$$

We may also note that if

$$
\int_{\Omega} \sigma \cdot D \zeta=-\int_{\Omega}(u-I) \zeta d x
$$

holds for arbitrary $\zeta \in B V(\Omega)$ for some $u$ where $\sigma$ is defined as above, then $u$ solves (1.1). In fact, for arbitrary $v \in B V(\Omega)$ we take $\zeta=v-u$, noting that by convexity of $\varphi$ we have $\varphi(\nabla v)-\varphi(\nabla u) \geq \nabla(v-u) \cdot \varphi_{P}(\nabla u)$ on $\Omega_{a}$, and that on $\Omega_{s}$ we have

$$
\int_{\Omega_{s}}\left|D^{s} v\right|-\int_{\Omega_{s}}\left|D^{s} u\right| \geq \int_{\Omega_{s}} D^{s}(v-u) \cdot \frac{D^{s} u}{\left|D^{s} u\right|} .
$$

The proof of the following lemma is based on [9], with some necessary modifications. 
LEMMA 2.7. Suppose $u$ is a solution to our minimization problem, $B_{2 r}(a) \subset \subset \Omega$ and $v \in C^{0,1}\left(B_{2 r}(a)\right)$ with $\sup _{B_{2 r}(a)}|\nabla v| \leq 1-\mu$, and

$$
\mathcal{L}^{n}\left(\{u \neq v\} \cap B_{\rho}(a)\right) \leq \frac{1}{2}\left|B_{\rho}\right| \quad \text { for all } \quad r \leq \rho \leq 2 r,
$$

then there exists positive constants $c_{9}$ and $c_{10}$ such that if

$$
\mathcal{L}^{n}\left(\{u \neq v\} \cap B_{2 r}(a)\right) \leq c_{9} r^{n}
$$

then

$$
\|u-v\|_{L^{\infty}\left(B_{r}(a)\right)} \leq c_{10}\left(\mathcal{L}^{n}\left(\{u \neq v\} \cap B_{2 r}(a)\right)\right)^{\frac{1}{n}}
$$

Proof. First we note that the function $\varphi$ satisfies $|p|-\lambda \leq \varphi(p) \leq|p|$ for all $p \in \mathbf{R}^{n}$, some $\lambda>0$. By convexity of $\varphi$ we have $\varphi(p) \leq \varphi_{P}(p) \cdot p+\varphi(0)$ for all $p \in \mathbf{R}^{n}$. Hence we have

$$
\begin{gathered}
|D u|=|\nabla u| d x+\left|D^{s} u\right| \leq \varphi(\nabla u) d x+\left|D^{s} u\right|+\lambda d x \\
\leq \varphi_{P}(\nabla u) \cdot \nabla u d x+\left|D^{s} u\right|+(\lambda+\varphi(0)) d x=\sigma \cdot D u+\lambda d x .
\end{gathered}
$$

Let $\theta: \mathbf{R} \rightarrow \mathbf{R}$ be a bounded, increasing, piecewise differentiable function with $\theta^{\prime}(t) \leq 1$ for almost all $t$. Let $0<\rho<h$ and

$$
\eta(x)= \begin{cases}1 & \text { in } B_{\rho}(a) \\ (h-\rho)^{-1}(h-|x-a|) & \text { in } B_{h}(a) \backslash B_{\rho}(a) \\ 0 & \text { in } \Omega \backslash B_{h}(a) .\end{cases}
$$

Now apply the first variational formula to $\zeta=\eta \theta(u-v)$ to get

$$
\begin{gathered}
\int_{B_{h}(a)} \eta \sigma \cdot D[\theta(u-v)]=(h-\rho)^{-1} \int_{B_{h}(a) \backslash B_{\rho}(a)} \sigma \cdot \frac{x-a}{|x-a|} \theta(u-v) d x \\
-\int_{B_{h}(a)} \eta \theta(u-v)(u-I) d x .
\end{gathered}
$$

In order to obtain a lower bound for $\eta \sigma \cdot D[\theta(u-v)]$ we use the above properties of $\varphi$. We have $D[\theta(u-v)]=\theta^{\prime}(u-v) D(u-v)$ and hence by noting the bound of $|\nabla v|$

$$
\begin{gathered}
\int_{B_{\rho}(a)}|D[\theta(u-v)]| \leq \int_{B_{\rho}(a)} \theta^{\prime}(u-v)|D u|+\int_{B_{\rho}(a)} \theta^{\prime}(u-v) \\
\quad \leq \int_{B_{\rho}(a)} \theta^{\prime}(u-v) \varphi(D u)+\int_{B_{\rho}(a)}(\lambda+1) \theta^{\prime}(u-v) \\
\leq \int_{B_{\rho}(a)} \theta^{\prime}(u-v) \sigma \cdot D u+\int_{B_{\rho}(a)}(\lambda+1) \theta^{\prime}(u-v)
\end{gathered}
$$




$$
=\int_{B_{\rho}(a)} \theta^{\prime}(u-v) \sigma \cdot D(u-v)+\int_{B_{\rho}(a)} \theta^{\prime}(u-v) \sigma \cdot D v
$$

$(2.23)+\int_{B_{\rho}(a)}(\lambda+1) \theta^{\prime}(u-v) \leq \int_{B_{h}(a)} \eta \sigma \cdot D[\theta(u-v)]+\int_{B_{h}(a)} C_{\lambda} \theta^{\prime}(u-v)$

for some constant $C_{\lambda}$ depending only on $\lambda$. Therefore, by inserting (2.22) into (2.23), and noting the $L^{\infty}$ bound for $u$, we get

$$
\begin{gathered}
\int_{B_{\rho}(a)}|D[\theta(u-v)]| \\
\leq(h-\rho)^{-1} \int_{B_{h}(a) \backslash B_{\rho}(a)}|\theta(u-v)| d x+C_{\lambda}|\operatorname{supp} \eta \theta(u-v)| \\
+2\|I\|_{L^{\infty}(\Omega)} \int_{B_{h}(a)}|\theta(u-v)| d x .
\end{gathered}
$$

Now for $0<k<s$ we choose $\theta$ as

$$
\theta(t)= \begin{cases}0 & \text { for } t \leq k \\ t-k & \text { for } k<t<s \\ s-k & \text { for } t \geq s .\end{cases}
$$

Now let $A(k, h) \equiv B_{h} \cap\{u-v>k\}$. Clearly supp $[\eta \theta(u-v)] \subset A(k, h)$. Thus

$$
\begin{gathered}
\int_{B_{\rho}(a)}|D[\theta(u-v)]| \\
\leq\left((h-\rho)^{-1}+2\|I\|_{L^{\infty}(\Omega)}\right) \int_{B_{h}(a)}|\theta(u-v)| d x+C_{\lambda}|A(k, h)|
\end{gathered}
$$

By assumption, $|A(0, \rho)| \leq \frac{1}{2}\left|B_{\rho}(a)\right|$ for $r \leq \rho \leq 2 r$. Thus we see that

$$
\frac{\mathcal{L}^{n}\left\{\{\theta(u-v)=0\} \cap B_{\rho}(a)\right\}}{\left|B_{\rho}(a)\right|} \geq \frac{1}{2} .
$$

We can then apply the isoperimetric inequality for $s>k>0$ to get

$$
\begin{gathered}
(s-k)|A(s, \rho)|^{\frac{n-1}{n}} \leq\left(\int_{B_{\rho}(a)}|\theta(u-v)|^{\frac{n}{n-1}} d x\right)^{\frac{n-1}{n}} \\
\leq c_{11} \int_{B_{\rho}(a)}|D[\theta(u-v)]|
\end{gathered}
$$




$$
\leq c_{12}\left((h-\rho)^{-1}+\|I\|_{L^{\infty}(\Omega)}\right) \int_{B_{h}(a)}|\theta(u-v)| d x+c_{13}|A(k, h)| .
$$

So since $h \leq 2 r$ we get

$$
(s-k)|A(s, \rho)|^{\frac{n-1}{n}} \leq c_{14}(h-p)^{-1} \int_{B_{h}(a)}|\theta(u-v)| d x+c_{14}|A(k, h)| .
$$

And since

$$
\int_{B_{h}(a)}|\theta(u-v)| d x \leq(s-k)|A(k, h)|
$$

we arrive at

$$
|A(s, \rho)|^{\frac{n-1}{n}} \leq c_{14}\left((h-p)^{-1}+(s-k)^{-1}\right)|A(k, h)|
$$

for every $r \leq \rho<h \leq 2 r$ and $s>k>0$. We now apply Lemma 2.1 in [9] to obtain the upper bound.

The lower bound for $u-v$ is obtained by using a similar argument for $0<k<$ $s<\infty$,

$$
\tilde{\theta}(t)= \begin{cases}0 & \text { for } t \geq-k \\ -t-k & \text { for }-s<t<-k \\ s-k & \text { for } t \leq-s\end{cases}
$$

and $\tilde{A}(k, h) \equiv B_{h} \cap\{u-v<-k\}$. The lemma then follows by again applying Lemma 2.1 in $[9]$.

Now define the energy function

$$
\begin{gathered}
\Phi(r, l, x)=\frac{1}{\left|B_{r}\right|}\left\{\int_{B_{r}(x) \cap\{|\nabla u| \geq 1\}}|\nabla u| d x\right. \\
\left.+\int_{B_{r}(x) \cap\{|\nabla u|<1\}}|\nabla u-l|^{2} d x+\int_{B_{r}(x)}\left|D^{s} u\right|\right\} .
\end{gathered}
$$

The following theorem provides a decay estimate for $\Phi$ :

ThEOREM 2.8. If $u$ solves (1.1) with $B_{r}(a) \subset \subset \Omega, l_{1} \in \mathbf{R}^{n}$ with $\left|l_{1}\right| \leq 1-\mu$, then there exist positive constants $\omega, \epsilon, \kappa, c_{37}, c_{38}$, and $c_{39}$ such that

$$
\Phi\left(4 r, l_{1}, a\right) \leq \epsilon
$$

and

$$
r \leq \kappa
$$

implies

$$
\Phi\left(\omega r, l_{2}, a\right) \leq \frac{1}{2} \Phi\left(4 r, l_{1}, a\right)+c_{37} r
$$


where

$$
\left|l_{1}-l_{2}\right| \leq c_{38} \Phi\left(4 r, l_{1}, a\right)^{\frac{1}{2}}+c_{39} r
$$

Proof. For fixed $\lambda>0$, define

$$
R^{\lambda} \equiv\left\{x \in B_{2 r}(a) \mid \Phi\left(\rho, l_{1}, x\right) \leq \lambda \text { for all } 0<\rho \leq 2 r\right\} .
$$

By Vitali's covering theorem, there exists disjoint balls $\left\{B_{r_{i}}\left(x_{i}\right)\right\}_{i=1}^{\infty}$ such that

$$
B_{2 r}(a) \backslash R^{\lambda} \subset \cup_{i=1}^{\infty} B_{5 r_{i}}\left(x_{i}\right)
$$

and $\Phi\left(r_{i}, l_{1}, x_{i}\right) \geq \lambda$. Then we have

$$
\mathcal{L}^{n}\left(B_{2 r}(a) \backslash R^{\lambda}\right) \leq 5^{n} \sum_{i=1}^{\infty}\left|B_{r_{i}}\left(x_{i}\right)\right| \leq \frac{5^{n}}{\lambda}\left|B_{4 r}(a)\right| \Phi\left(4 r, l_{1}, a\right) .
$$

Let $g(x)=u(x)-l_{1} \cdot x$. By Poincare's inequality we have for $x \in R^{\lambda}$ and $0<\rho \leq 2 r$

$$
\begin{gathered}
\quad \frac{1}{\left|B_{\rho}\right|} \int_{B_{\rho}(x)}\left|g(y)-\bar{g}_{x, \rho}\right| d y \leq \frac{c_{15}}{\rho^{n-1}} \int_{B_{\rho}(x)}|D g| \\
\leq \frac{c_{15}}{\rho^{n-1}}\left\{2 \int_{B_{\rho}(x) \cap\{|\nabla u| \geq 1\}}|\nabla u| d x+\int_{B_{\rho}(x)}\left|D^{s} u\right|\right. \\
\left.+\left|B_{\rho}\right|^{1 / 2}\left(\int_{B_{\rho}(x) \cap\{|\nabla u|<1\}}\left|\nabla u-l_{1}\right|^{2} d x\right)^{1 / 2}\right\} \\
\leq c_{16} \rho \Phi\left(\rho, l_{1}, x\right)^{1 / 2} \leq c_{16} \lambda^{1 / 2} \rho
\end{gathered}
$$

where $\bar{g}_{x, \rho}=\frac{1}{\left|B_{\rho}\right|} \int_{B_{\rho}(x)} g(y) d y$. Then

$$
\begin{gathered}
\left|\bar{g}_{x, \rho / 2^{k+1}}-\bar{g}_{x, \rho / 2^{k}}\right| \leq \frac{1}{\left|B_{\rho / 2^{k+1}}\right|} \int_{B_{\rho / 2^{k+1}}(x)}\left|g(y)-\bar{g}_{x, \rho / 2^{k}}\right| d y \\
\leq 2^{n} \frac{1}{\left|B_{\rho / 2^{k}}\right|} \int_{B_{\rho / 2^{k}}(x)}\left|g(y)-\bar{g}_{x, \rho / 2^{k}}\right| d y \leq c_{17} \rho \lambda^{1 / 2} / 2^{k} .
\end{gathered}
$$

Since $g(x)=\lim _{\rho \rightarrow 0} \bar{g}_{x, \rho}$ for $\mathcal{L}^{n}$ a.e. $x \in R^{\lambda}$,

$$
\left|g(x)-\bar{g}_{x, \rho}\right| \leq \sum_{k=1}^{\infty}\left|\bar{g}_{x, \rho / 2^{k+1}}-\bar{g}_{x, \rho / 2^{k}}\right| \leq c_{17} \rho \lambda^{1 / 2}
$$

For $x, y \in R^{\lambda}$ with $|x-y| \leq 2 r$, set $\rho=|x-y|$. Then

$$
\left|\bar{g}_{x, \rho}-\bar{g}_{y, \rho}\right| \leq \frac{1}{\left|B_{\rho}(x) \cap B_{\rho}(y)\right|} \int_{B_{\rho}(x) \cap B_{\rho}(y)}\left|\bar{g}_{x, \rho}-g(z)\right|+\left|g(z)-\bar{g}_{y, \rho}\right| d z
$$




$$
\leq c_{18} \frac{1}{B_{\rho}}\left(\int_{B_{\rho}(x)}\left|g(z)-\bar{g}_{x, \rho}\right| d z+\int_{B_{\rho}(y)}\left|g(z)-\bar{g}_{y, \rho}\right| d z\right) \leq c_{19} \lambda^{1 / 2} \rho .
$$

So by combining the above, we have

$$
|g(x)-g(y)| \leq c_{20} \lambda^{1 / 2} \rho=c_{20} \lambda^{1 / 2}|x-y|
$$

for $\mathcal{L}^{n}$ a.e. $x, y \in R^{\lambda} \subset B_{2 r}(a)$. Let $\lambda=c_{20}^{-2} \beta^{4 \delta}$, so that

$$
\left|u(x)-l_{1} \cdot x-u(y)+l_{1} \cdot y\right|=|g(x)-g(y)| \leq \beta^{2 \delta}|x-y|,
$$

and let $v$ be a Lipschitz function defined on $B_{2 r}(a)$ such that

$$
v=u \text { on } R^{\lambda} \text {, and } \sup _{B_{2 r}(a)}\left|\nabla v-l_{1}\right| \leq \beta^{2 \delta} .
$$

Such a $v$ exists by a standard extension for a Lipschitz function. Also note that for this choice of $v$ we have $\sup _{B_{2 r}(a)}|v| \leq C_{u}$. With this choice of $\lambda$, and by choosing

$$
\beta=\Phi\left(4 r, l_{1}, a\right) \text { and } \delta=\frac{1}{8(n+1)},
$$

we can estimate the size of the non-zero set of $u-v$ as

$$
\mathcal{L}^{n}\left(B_{2 r}(a) \cap\{u \neq v\}\right) \leq c_{21} r^{n} \beta^{-4 \delta} \Phi\left(4 r, l_{1}, a\right) \leq c_{21} r^{n} \Phi\left(4 r, l_{1}, a\right)^{1-4 \delta} .
$$

We made the choice of $\delta$ so that $(1-4 \delta) \cdot \frac{n+1}{n}=1+\frac{1}{2 n}>1$. Now choose $\tilde{r} \in\left[\frac{1}{2} r, r\right]$ so that both

$$
\int_{\partial B_{\tilde{r}(a)}}|u-v| d \mathcal{H}^{n-1} \leq \frac{3}{r} \int_{B_{\tilde{r}(a)}}|u-v| d x
$$

and

$$
\int_{\partial B_{\tilde{r}(a)}}\left|u-\bar{u}_{a, r}-l_{1} \cdot(x-a)\right| d \mathcal{H}^{n-1} \leq \frac{3}{r} \int_{B_{\tilde{r}(a)}}\left|u-\bar{u}_{a, r}-l_{1} \cdot(x-a)\right| d x
$$

are satisfied. By the choice of $\tilde{r}$,

$$
\int_{\partial B_{\tilde{r}(a)}}|u-v| d \mathcal{H}^{n-1} \leq \frac{3}{r}\|u-v\|_{L^{\infty}\left(B_{r}(a)\right)} \cdot \mathcal{L}^{n}\left(B_{r}(a) \cap\{u \neq v\}\right) .
$$

Choose $r\left(C_{u}+\|I\|_{L^{\infty}(\Omega)}\right) \leq c_{6}$. By Lemma 2.7, for $\Phi\left(4 r, l_{1}, a\right) \leq c_{22}$, we have

$$
\frac{1}{r}\|u-v\|_{L^{\infty}\left(B_{r}(a)\right)} \leq c_{10} \frac{1}{r}\left(\mathcal{L}^{n}\left(B_{2 r}(a) \cap\{u \neq v\}\right)\right)^{1 / n} .
$$

Thus

$$
\frac{1}{r^{n}} \int_{\partial B_{\tilde{r}(a)}}|u-v| d \mathcal{H}^{n-1} \leq c_{23} \Phi\left(4 r, l_{1}, a\right)^{1+\frac{1}{2 n}} .
$$

We now apply Lemma 2.6 to the above, using the estimate for the boundary integral of $u-v$, to obtain

$$
(2.25) \int_{B_{r \omega}(a)}\left|D^{s} u\right|+\int_{B_{r \omega(a)} \cap\{|\nabla u| \geq 1\}}|\nabla u| d x+\int_{B_{r \omega(a)} \cap\{|\nabla u|<1\}}|\nabla(u-w)|^{2} d x
$$




$$
\leq c_{24} r^{n}\left(\Phi\left(4 r, l_{1}, a\right)^{1+\frac{1}{2 n}}+\Phi\left(4 r, l_{1}, a\right)^{1+\frac{1}{4(n+2)}}\right)
$$

for any $\omega \leq 1 / 2$. Let $l_{2} \equiv \nabla \omega(a)$. By using the gradient estimate, (2.7)-(2.8), for $\omega$, the choice of $\tilde{r}$, the definition of $v_{\beta}$, the above bound for $v$, and Poincare's inequality,

$$
\begin{gathered}
\left|l_{1}-l_{2}\right| \leq \frac{1}{\left|B_{\tilde{r}}\right|} \int_{\partial B_{\tilde{r}(a)}}\left|v_{\beta}-\bar{u}_{a, r}-l_{1} \cdot(x-a)\right| d \mathcal{H}^{n-1}+c_{25} r\left(\|I\|_{L^{\infty}(\Omega)}+C_{u}\right) \\
\leq \frac{1}{\left|B_{\tilde{r}}\right|} \int_{\partial B_{\tilde{r}(a)}}\left|v_{\beta}-u\right|+\left|u-\bar{u}_{a, r}-l_{1} \cdot(x-a)\right| d \mathcal{H}^{n-1}+c_{25} r\left(\|I\|_{L^{\infty}(\Omega)}+C_{u}\right) \\
\leq c_{26} \Phi\left(4 r, l_{1}, a\right)+\frac{c_{27}}{r^{n}} \int_{B_{r}(a)}\left|D u-l_{1}\right|+c_{25} r\left(\|I\|_{L^{\infty}(\Omega)}+C_{u}\right) .
\end{gathered}
$$

By the Hölder inequality, we obtain $\left|l_{1}-l_{2}\right| \leq c_{28} \Phi\left(4 r, l_{1}, a\right)^{1 / 2}+c_{25} r\left(\|I\|_{L^{\infty}(\Omega)}+C_{u}\right)$. The last term on the left side of inequality (2.25) satisfies

$$
\int_{B_{r \omega}(a) \cap\{|\nabla u|<1\}}|\nabla(u-w)|^{2} d x \geq \int_{B_{r \omega}(a) \cap\{|\nabla u|<1\}} \frac{1}{2}\left|\nabla u-l_{2}\right|^{2}-\left|\nabla w-l_{2}\right|^{2} d x .
$$

Thus by (2.25) and the above inequality,

$$
\left|B_{r \omega}\right| \Phi\left(r \omega, l_{2}, a\right) \leq c_{29} r^{n} \Phi\left(4 r, l_{1}, a\right)^{1+\frac{1}{4(n+2)}}+c_{30} \int_{B_{r \omega}}\left|\nabla w-l_{2}\right|^{2} d x .
$$

To estimate the last term, we again use the estimates for the gradient of $w$. Note that

$$
\begin{aligned}
& \sup _{x, y \in B_{r / 4}(a)} \frac{|\nabla w(x)-\nabla w(y)|}{|x-y|^{1 / 2}} \\
& \leq c_{4} \frac{1}{r^{n+1 / 2}} \int_{\partial B_{\tilde{r}(a)}}\left|v_{\beta}-\bar{u}_{a, r}-l_{1} \cdot(x-a)\right| d \mathcal{H}^{n-1} \\
& +c_{4} r^{1 / 2}\left(\|I\|_{L^{\infty}(\Omega)}+C_{u}\right) .
\end{aligned}
$$

Thus, similar to the estimate for $\left|l_{1}-l_{2}\right|$, we have

$$
\begin{aligned}
& \sup _{x, y \in B_{r / 4}(a)} \frac{|\nabla w(x)-\nabla w(y)|}{|x-y|^{1 / 2}} \\
& \leq c_{31}\left(r^{-1 / 2} \Phi\left(4 r, l_{1}, a\right)^{1 / 2}+r^{1 / 2}\left(\|I\|_{L^{\infty}(\Omega)}+C_{u}\right) .\right.
\end{aligned}
$$

Using this we then have

$$
\begin{gathered}
\int_{B_{r \omega}(a)}\left|\nabla w-l_{2}\right|^{2} d x \leq c_{32}(r \omega)^{n}\left\{\omega \Phi\left(4 r, l_{1}, a\right)\right. \\
\left.+r \Phi\left(4 r, l_{1}, a\right)^{1 / 2}\left(\|I\|_{L^{\infty}(\Omega)}+C_{u}\right)+r^{2}\left(\|I\|_{L^{\infty}(\Omega)}+C_{u}\right)^{2}\right\} \\
\leq c_{33}(r \omega)^{n}\left\{\omega \Phi\left(4 r, l_{1}, a\right)+r\left(\|I\|_{L^{\infty}(\Omega)}+C_{u}\right)\right\} .
\end{gathered}
$$


Hence by combining the above with (2.26) we have

$$
\begin{aligned}
\Phi\left(r \omega, l_{2}, a\right) & \leq c_{34} \omega^{-n} \Phi\left(4 r, l_{1}, a\right)^{1+\frac{1}{4(n+1)}}+c_{35} \omega \Phi\left(4 r, l_{1}, a\right) \\
& +c_{36} r\left(\|I\|_{L^{\infty}(\Omega)}+C_{u}\right) .
\end{aligned}
$$

Choose $\omega<1 / 4$ so small so that $c_{35} \omega<1 / 4$, and again restrict $\Phi\left(4 r, l_{1}, a\right)$ so that $c_{34} \omega^{-n} \Phi\left(4 r, l_{1}, a\right)^{1+\frac{1}{4(n+1)}}<1 / 4$. This now proves the theorem. $[1]$.

We now prove Theorem 1.1 using and iteration argument (see for example [10] or

Proof. Assume that $\frac{1}{\left|B_{r}\right|} \int_{B_{r}(a)}\left|D u-l_{1}\right| \leq \epsilon_{0}$ for some $l_{1} \in \mathbf{R}^{n}$ with $\left|l_{1}\right| \leq 1-4 \mu$ and for any $r$ with $r \leq \kappa$. For each $x \in B_{r / 2}(a)$ we have

$$
\Phi\left(r / 2, l_{1}, x\right) \leq 2^{n} \Phi\left(r, l_{1}, a\right) \leq c_{40} \frac{1}{B_{r}} \int B_{r}(a)\left|D u-l_{1}\right| \leq c_{40} \epsilon_{0} .
$$

We will use Theorem 2.8 iteratively. Choose $\epsilon_{0}$ so small so that $c_{40} \epsilon_{0} \leq \epsilon$ and restrict $r$ so that $c_{37} r \leq r / 2$. Assume $\left|l_{j-1}\right|<1-2 \mu$ and

$$
\begin{gathered}
\Phi\left(\left(\frac{\omega}{4}\right)^{j-1} \frac{r}{2}, l_{j}, x\right) \leq\left(\frac{1}{2}\right)^{j-1} \Phi\left(\frac{r}{2}, l_{1}, x\right) \\
+\sum_{i=1}^{j-1}\left(\frac{1}{2}\right)^{j-1} \omega^{j-i-1} c_{41} r \text { for } j=2, \ldots, k
\end{gathered}
$$

We need to show $\Phi\left(\left(\frac{\omega}{4}\right)^{k-1} \frac{r}{2}, l_{k}, x\right) \leq \epsilon$ and $\left|l_{k}\right|<1-2 \mu$. To continue the inductive step. Since $\omega<1 / 2$,

$$
\sum_{i=1}^{k-1}\left(\frac{1}{2}\right)^{i-1} \omega^{k-i-1} \leq\left(\frac{1}{2}\right)^{k-2}(k-1) \leq c_{42}\left(\frac{1}{2}\right)^{k / 2}
$$

for all $k$. By further restricting $r$, we have

$$
\Phi\left(\left(\frac{\omega}{4}\right)^{k-1} \frac{r}{2}, l_{k}, x\right) \leq \epsilon
$$

Note that

$$
\begin{gathered}
\left|l_{k}\right| \leq \sum_{j=1}^{k-1}\left|l_{j+1}-l_{j}\right|+\left|l_{1}\right| \\
\leq \sum_{j=1}^{k-1}\left\{c_{38} \Phi\left(\left(\frac{\omega}{4}\right)^{j-1} \frac{r}{2}, l_{j}, x\right)^{1 / 2}+c_{39}\left(\frac{\omega}{4}\right)^{j-1} r\right\}+1-4 \mu \\
\leq c_{38} \sum_{j=1}^{k-1}\left\{\left(\frac{1}{2}\right)^{(j-1) / 2} \Phi\left(\frac{r}{2}, l_{1}, x\right)^{1 / 2}+c_{41}^{1 / 2}\left(\frac{1}{2}\right)^{j / 4} c_{37}^{1 / 2} r^{1 / 2}\right\}
\end{gathered}
$$




$$
\begin{gathered}
+c_{39} r \sum_{j=1}^{k-1}\left(\frac{\omega}{4}\right)^{j-1}+1-4 \mu \\
\leq c_{42} \Phi\left(\frac{r}{2}, l_{1}, x\right)^{1 / 2}+c_{42} r^{1 / 2}+1-4 \mu .
\end{gathered}
$$

Hence by restricting $\epsilon_{0}$ and $r$ again, we can see that $\left|l_{k}\right|<1-2 \mu$. Thus may continue the iterative step indefinitely, giving

$$
\lim _{k \rightarrow \infty}\left(\Phi\left(\frac{\omega}{4}\right)^{k} \frac{r}{2}, l_{k+1}, x\right)=0 \text { for all } x \in B_{r / 2}(a) .
$$

Thus

$$
\lim _{\rho \rightarrow 0} \frac{1}{\left|B_{\rho}\right|}\left(\int_{B_{\rho}(x)}\left|D^{s} u\right|+\int_{B_{\rho}(x) \cap\{|\nabla u| \geq 1\}}|\nabla u| d x\right)=0
$$

for all $x \in B_{r / 2}(a)$. We see that (see for instance [5])

$$
\left|D^{s} u\right|\left(B_{r / 2}(a)\right)=0
$$

with $|\nabla u| \leq 1-\mu<1$ a.e on $B_{r / 2}(a)$. By (2.21), $u$ also satisfies the stated equation. $\square$

¿From this, we easily prove Theorem 1.2 .

Proof. Assume that $u$ is a minimizer of (1.1) and that $\tilde{E}=\{|\nabla u|<1\}$ has positive Lebesgue measure. ¿From standard measure theory (see for example [5]) we have

$$
\lim _{r \rightarrow 0} \frac{1}{\left|B_{r}\right|} \int_{B_{r}(x)}\left|D^{s} u\right|=0
$$

for $\mathcal{L}^{n}$-a.e. $x \in \tilde{E}$. Also, since $|\nabla u| \in L^{1}(\Omega)$,

$$
\lim _{r \rightarrow 0} \frac{1}{\left|B_{r}\right|} \int_{B_{r}(x)}|\nabla u(y)-\nabla u(x)| d x=0
$$

for $\mathcal{L}^{n}$-a.e. $x \in \tilde{E}$ Lebesgue's differentiation theorem. Now let $E$ be the set of all points of $\tilde{E}$ for which either one of the above does not hold. Clearly $\mathcal{L}^{n}(\tilde{E} \backslash E)=0$, $|\nabla u|<1$ on $E$, and both (2.27) and (2.28) hold at each point of $E$. For each fixed $x \in E$, there exists some $\mu_{x}>0$ such that

$$
|\nabla u(x)|<1-2 \mu_{x} .
$$

Then (2.27) and (2.28) combined with Theorem 1.1 show that there exists an $r_{x}$ such that

$$
\left|D^{s} u\right|\left(B_{r_{x}}(x)\right)=0 \text { and }|\nabla u|<1-\mu_{x} \text { on } B_{r_{x}}(x)
$$

and $u \in C^{1, \alpha}\left(B_{r_{x}}(x)\right)$, giving $B_{r_{x}}(x) \subset E$ in particular. Thus $E$ is an open set in $\Omega$ with the required properties. 


\section{REFERENCES}

[1] G. Anzellotti, M. Giaquinta, Convex functionals and partial regularity, Arch. Rat. Mech. Anal. 102 (1988), pp. 243-272.

[2] P. Blomgren and T.Chan, Color TV: Total variation methods for restoration of vector-valued images, IEEE Tran. Image Processing, Vol. 7 (3), (1998), pp. 304-309.

[3] A. Chambolle, P.L. Lions, Image recovery via total variation minimization and related problems, Numerische Mathematik 76 (1997), pp. 167-188.

[4] I. Ekeland, R. Temam, Convex analysis and variational problems, North Holland, Amsterdam (1976).

[5] L. Evans, R. Gariepy, Measure theory and fine properties of functions, CRC Press, Boca Raton (1992).

[6] M. Giaquinta, Introduction to regularity Theory for nonlinear elliptic systems, Birkhauser, (1993).

[7] D. Gilbarg, N. Trudinger, Elliptic partial differential equations of second order, 2nd ed., Springer-Verlag (1983).

[8] R. Hardt, D. Kinderlehrer, Elastic plastic deformation, Appl. Math. Optim. 10 (1983), pp. 203-246.

[9] R. HARDT, D. Kinderlehrer, Variational problems with linear growth, PDEs and Cal. Var., Vol. 2, Birkhauser (1989), pp. 633-659.

[10] R. Hardt, Y. Tonegawa, Partial regularity for evolution problems with discontinuity, Manuscripta Math. 90 (1996), no. 1, pp. 85-103.

[11] R. Hardt, X. Zhou, An evolution problem for linear growth functionals, Commun. in Partial Differential Equations 19(11\&12) (1994), pp. 1879-1907.

[12] L. Rudin And S. Osher, Total variation based image restoration with free local constraints, Proc. of IEEE ICIP-94, Austin, TX, Vol.1, (1994), pp. 31-35.

[13] L. Rudin, S. Osher, AND E. FAtemi, Nonlinear total variation based noise removal algorithms, Physica D 60, North-Holland (1992), pp. 259-268.

[14] R. Schoen, L. Simon, A new proof of the regularity theorem for rectifiable currents which minimize parametric elliptic functionals, Indiana Math. J. 31(3) (1982), pp. 415-434.

[15] D. Strong and T. Chan, Edge-preserving and scale-dependent properties of total variation Regularization, CAM00-38(UCLA).

[16] X. ZHOU, An evolution problem for plastic antiplanar shear, Appl. Math. Optim. 25 (1992), pp. 263-285. 